\title{
TsakonasA new reactive sputtering technique for the low temperature deposition of transparent light emitting ZnS:Mn thin films
}

\section{Steve Wakeham ${ }^{*}, 1$ Mike Thwaites ${ }^{1}$, Costas Tsakonas ${ }^{2}$, Wayne Cranton ${ }^{2}$, Robert Ranson ${ }^{2}$, Gabriel Bou- taud $^{2}$ and Demosthenes Koutsogeorgis ${ }^{2}$}

${ }^{1}$ Plasma Quest Limited, Unit 1B Rose Estate, Osborn Way, Hook, UK

${ }^{2}$ Nottingham Trent University, School of Science and Technology, Clifton lane, Nottingham, NG11 8NS, UK

Received ZZZ, revised ZZZ, accepted ZZZ

Published online ZZZ (Dates will be provided by the publisher.)

PACS 81.15.Cd, 81.15.Ef, 42.79.Kr, 52.50.Dg, 78.20.-e

* Steve Wakeham: e-mail steve.wakeham@plasmaquest.co.uk, Phone: +44 (0)1256 740 680, Fax: +44 (0)1256 740690

\begin{abstract}
The temperature sensitive nature of the substrates used in the flexible displays market necessitates a low temperature deposition technique for processing them. ZnS:Mn exhibiting high intensity photoluminescence and good crystallinity has been deposited onto $\mathrm{Si}$ wafers, glass microscope slides and polymeric substrates using a new reactive sputtering technology referred to as HiTUS. This technique enables very high deposition
\end{abstract}

1 Introduction This work compares two different sputtering techniques for the deposition of alternating current thin film electroluminescent (ACTFEL) devices. High target utilisation sputtering (HiTUS) is a new method for sputtering thin films that allows for the deposition of working electroluminescent (EL) structures with no substrate heating or post deposition annealing [1]. However, here we look at the effect of post deposition annealing HiTUS devices that have been deposited onto silicon and glass substrates with no substrate heating. Radio frequency (RF) magnetron sputtering has also been used for the fabrication of comparable devices with both substrate heating and post deposition annealing. The results of quantitative EL measurements for structures deposited using these two techniques are presented here.

2 Experimental HiTUS and RF magnetron sputtering have both been used to deposit devices with the double insulating layer structure incorporating $\mathrm{ZnS}: \mathrm{Mn}$ as the active, light emitting layer, $\mathrm{Y}_{2} \mathrm{O}_{3}$ as the dielectric layers and ITO as the front and back contact electrodes.

\begin{abstract}
rates and requires no substrate heating. When incorporated as part of a complete EL device, as-deposited ZnS:Mn films are seen to exhibit stable electroluminescence on $\mathrm{Si}$, glass and planarised PET substrate materials. Post annealing of the devices on Si and glass at temperatures of up to $600{ }^{\circ} \mathrm{C}$ show that the HiTUS films perform better than equivalent $\mathrm{ZnS}$ :Mn films deposited using $\mathrm{RF}$ magnetron sputtering.
\end{abstract}

2.1 High Target Utilisation Sputtering Unlike RF magnetron sputtering, HiTUS relies on the generation of a plasma remotely from the targets. An RF coil antennae surrounding a quartz glass tube is located in a side arm (referred to as the plasma launch system, or PLS) adjacent to the vacuum chamber. The plasma is initiated here and amplified by a launch electromagnet at the exit of the PLS. A steering electromagnet is then used to focus and control the direction of the plasma. This system uses similar principles to an RF inductively coupled plasma but the magnetic enhancement provided by HiTUS results in higher plasma densities, of the order of $10^{13}$ ions $\mathrm{cm}^{-3}$. Further details are described elsewhere [2]. With careful control of the current flowing through each of the electromagnets, the plasma beam can be directed such as to cover the full surface area of the target. With the application of a sufficient negative bias, argon ions are then accelerated into the target resulting in a high current density over the full surface area of the target. Hence the target is uniformly eroded resulting in a significant reduction in target poisoning over conventional magnetron sputtering and hence also a much im- 
proved deposition rate when depositing non-metallic thin films.

The HiTUS system used for this work accommodates four 4" diameter, $6 \mathrm{~mm}$ thick water cooled targets. This allows for the deposition of complete devices without needing to break the vacuum and potentially introduce contaminants.

2.1.2 Deposition of ITO, $\mathrm{Y}_{2} \mathrm{O}_{3}$ and $\mathrm{ZnS}: \mathrm{Mn}$ using HiTUS All the materials used in this work were deposited from metallic targets in a reactive gas mixture of argon and either oxygen or hydrogen sulphide. Prior to depositing each layer, the substrates were immersed in a diffuse, high density plasma. This is easily achieved using HiTUS by switching off the steering electromagnet. The impinging low energy (less than $50 \mathrm{eV}$ ) argon ions remove volatile species from the substrate surface helping to promote adhesion of subsequent layers. It is the intension to also investigate the effect this diffuse plasma could have on the interface states between the $\mathrm{ZnS}: \mathrm{Mn}$ and dielectric layers.

After this substrate pre-conditioning, the target is sputter cleaned for up to 5 minutes prior to admission of the reactive gas. Deposition conditions are then allowed to stabilise for a further 5 minutes before the shutter, which is located immediately adjacent to the substrates, is opened and deposition commences.

Other than the devices deposited onto silicon wafers, an ITO back contact was used. This process has been previously developed to be compatible with polymeric substrate materials and hence is a low temperature process. Details can be found elsewhere [2].

Both the $\mathrm{Y}_{2} \mathrm{O}_{3}$ and the $\mathrm{ZnS}: \mathrm{Mn}$ were also deposited with no substrate heating, making this procedure directly transferable to plastics. A summary of the deposition conditions used for each of the materials within the EL structure is given in table 1 .

Table 1 HiTUS deposition parameters.

\begin{tabular}{cccccc}
\hline Material & $\begin{array}{c}\text { Process } \\
\text { pressure } \\
(\mathrm{mbar})\end{array}$ & $\begin{array}{c}\text { RF power } \\
(\mathrm{PLS}) \\
(\mathrm{kW})\end{array}$ & $\begin{array}{c}\text { DC power } \\
(\text { target }) \\
(\mathrm{kW})\end{array}$ & $\begin{array}{c}\text { Sub. Temp. } \\
\left({ }^{\circ} \mathrm{C}\right)\end{array}$ & $\begin{array}{c}\text { Dep. Rate } \\
(\mathrm{nm} / \mathrm{min})\end{array}$ \\
\hline $\mathrm{ITO}$ & $3.6 \times 10^{-3}$ & 1.0 & 0.81 & $<70{ }^{\circ} \mathrm{C}$ & 87 \\
$\mathrm{Y}_{2} \mathrm{O}_{3}$ & $4.0 \times 10^{-3}$ & 1.99 & 1.50 & $<70^{\circ} \mathrm{C}$ & 28 \\
$\mathrm{ZnS} \mathrm{Mn}$ & $7.7 \times 10^{-3}$ & 0.79 & 0.20 & $<70^{\circ} \mathrm{C}$ & 52 \\
\hline
\end{tabular}

2.2 RF magnetron sputtering For a comparative study, two separate RF magnetron sputtering systems $(13.56 \mathrm{MHz})$ were also used for the deposition of $\mathrm{Y}_{2} \mathrm{O}_{3}$, $\mathrm{ZnS}: \mathrm{Mn}$ and ITO. A base pressure of $1.2 \times 10^{-6} \mathrm{mbar}$ was used for the $\mathrm{Y}_{2} \mathrm{O}_{3}$ and $\mathrm{ZnS}: \mathrm{Mn}$ layers and the second system used to deposit the ITO was evacuated to a base pressure of $1.6 \times 10^{-7}$ mbar. Diffusion pumps backed by rotary pumps were used to attain this vacuum in both plants. The substrates were loaded into the main chamber through a side load lock. Two RF magnetron guns were positioned at $30^{\circ}$ to the vertical and $15 \mathrm{~cm}$ from the substrate surface for the dielectric/phosphor depositions. The same configuration was used for the ITO coatings but with a targetsubstrate separation of $10 \mathrm{~cm}$. The two main chambers were equipped with mass flow controlled gas lines providing $100 \%$ Argon for the EL device layers (dielectric and phosphor films) and $0.2 \%$ Oxygen/Argon for the ITO contacts. Alkali-free borosilicate glass and n-type silicon substrates were used in this study. Prior to deposition the glass and $\mathrm{Si}$ wafers were baked at $350{ }^{\circ} \mathrm{C}$ and $500{ }^{\circ} \mathrm{C}$ respectively. All targets were pre-sputtered for between 2 and 5 minutes prior to deposition. The $\mathrm{ZnS}: \mathrm{Mn}$ layers were deposited by co-sputtering a $99.99 \%$ pure $\mathrm{ZnS}$ target and a $99.95 \%$ pure $\mathrm{ZnS}$ target doped with $1.0 \mathrm{wt}$ \% Mn. Two RF power supplies providing $80 \mathrm{~W}$ to the $\mathrm{ZnS}$ target and $40 \mathrm{~W}$ to the target doped with $\mathrm{Mn}$ gave $\mathrm{ZnS}$ thin films with 0.5 wt. $\% \mathrm{Mn}$. The $\mathrm{Y}_{2} \mathrm{O}_{3}$ and ITO layers were deposited from $99.99 \%$ pure compound targets. All targets were 3" in diameter and provided by Testbourne UK. Table 2 shows the growth parameters for all layers (the total power on both targets is shown for the $\mathrm{ZnS}: \mathrm{Mn}$ layer).

Table 2 RF magnetron sputtering deposition parameters.

\begin{tabular}{ccccc}
\hline Material & $\begin{array}{c}\text { Process } \\
\text { pressure } \\
(\text { mbar })\end{array}$ & $\begin{array}{c}\text { RF power } \\
(\mathrm{W})\end{array}$ & $\begin{array}{c}\text { Sub. Temp. } \\
\left({ }^{\circ} \mathrm{C}\right)\end{array}$ & $\begin{array}{c}\text { Dep. Rate } \\
(\mathrm{nm} / \mathrm{min})\end{array}$ \\
\hline $\mathrm{ITO}$ & $2.66 \times 10^{-3}$ & 50 & $180{ }^{\circ} \mathrm{C}$ & 4 \\
$\mathrm{Y}_{2} \mathrm{O}_{3}$ & $6.66 \times 10^{-3}$ & 120 & $200^{\circ} \mathrm{C}$ & 1.5 \\
$\mathrm{ZnS}: \mathrm{Mn}$ & $6.66 \times 10^{-3}$ & 120 & $200^{\circ} \mathrm{C}$ & 3.7 \\
\hline
\end{tabular}

2.3 Comparison of sputtering methods The primary difference between the two sputtering techniques described above is that HiTUS decouples the ion density, which is controlled by the RF antennae power supply, from the ion energy, which is controlled by the DC bias applied to the target. This offers greater control over the growth process due to the independent control of multiple sputtering parameters.

From tables 1 and 2 it is clear that the HiTUS system also enables growth rates in excess of an order of magnitude faster than those offered by RF magnetron sputtering. Whilst higher deposition rates can be achieved using magnetron sputtering, this is to the detriment of the film properties since the RF power must be increased, with concomitant increase of the target bias, resulting in damage to film growth due to high energy ion bombardment $[3,4]$. Also of note is that these high growth rates are achieved using no substrate heating hence making HiTUS ideal for applications that require plastic substrates.

2.4 PL and EL measurement facility The photoluminescence (PL) measurements were carried out in a dark room at ambient temperature using a Laser Science VSL-337 ND nitrogen laser operating at $337 \mathrm{~nm}$ and $4 \mathrm{~ns}$ 
pulses. A power of $400 \mu \mathrm{J} /$ pulse with a $20 \mathrm{~Hz}$ repetition rate was used. The intensity of the PL emission was measured using an S2000 Ocean Optics fibre optic spectrometer.

The EL measurements were also carried out in a dark room. A TTi TG1010 programmable function generator, a voltage amplifier (maximum supply of $900 \mathrm{~V}$ peak to peak) and a Minolta LS-110 luminance meter were used to drive the devices and record their luminance. The input devices were all connected in series to a current limiting $100 \mathrm{k} \Omega$ resistor. All EL measurements were carried out using a sinusoidal waveform voltage at a frequency of $1 \mathrm{kHz}$ with voltage increments of $8 \mathrm{~V}$ peak to peak and $5 \mathrm{~s}$ duration.

3 Photoluminescence results Prior to making a complete electroluminescent device using $\mathrm{ZnS}: \mathrm{Mn}$ as the active, light emitting layer, individual films were first optimized for PL.

Three different targets were used to investigate the effect of different Mn concentrations on the PL properties of ZnS:Mn thin films deposited using HiTUS. Single layers of $\mathrm{ZnS}: \mathrm{Mn}$ with thicknesses of between 0.5 and $1.0 \mu \mathrm{m}$ were initially deposited at room temperature onto glass microscope slides from $\mathrm{Zn}$ targets doped with $0.4,0.5$ and 0.6 wt.\% Mn. For each of the three $\mathrm{Zn}: \mathrm{Mn}$ targets the $\mathrm{H}_{2} \mathrm{~S}$ flow rate was optimized to produce films that exhibited intense PL when excited by a nitrogen gas laser. As shown in Figure 1, the $\mathrm{Zn}$ target doped with $0.6 \mathrm{wt}$.\% Mn consistently gave films with the highest PL intensity. The peak intensity of the emitted light is centred at approximately $600 \mathrm{~nm}$, consistent with the 580 to $590 \mathrm{~nm}$ characteristic emission of Mn phosphor.

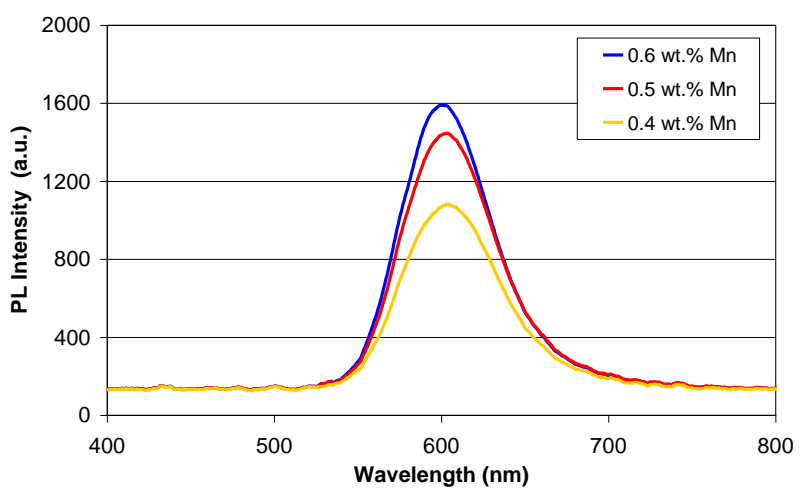

Figure 1 HiTUS results showing luminous intensity as a function of wavelength for $\mathrm{ZnS}$ :Mn films optimised with different concentrations of $\mathrm{Mn}$ in the target.

By increasing the $\mathrm{H}_{2} \mathrm{~S}$ flow rate during deposition the coatings are seen to become fully transparent. For optimized, fully transparent films, the deposition rate can be increased to $90 \mathrm{~nm} / \mathrm{min}$ for a target power of $0.5 \mathrm{~kW}$. However, whilst this high deposition rate did produce coatings that displayed intense PL, they exhibited very poor breakdown properties when incorporated as part of an EL structure. ZnS:Mn films produced at lower powers were seen to exhibit excellent PL and EL properties. Figure 2 shows that for optimum PL a target power of approximately $200 \mathrm{~W}$ should be used, giving a deposition rate of 52 $\mathrm{nm} / \mathrm{min}$. The results of optimised $\mathrm{ZnS}: \mathrm{Mn}$ films deposited at $200{ }^{\circ} \mathrm{C}$ using $\mathrm{RF}$ magnetron sputtering have also been included for comparison. Annealing these films at $500{ }^{\circ} \mathrm{C}$ sees an increase in the PL intensity but they still do not emit as brightly as those deposited using HiTUS. The HiTUS films were deposited with no substrate heating or post deposition annealing. Whilst the film deposited at $200 \mathrm{~W}$ was slightly thicker than the others, this only accounts for a small proportion of the increase in PL intensity.

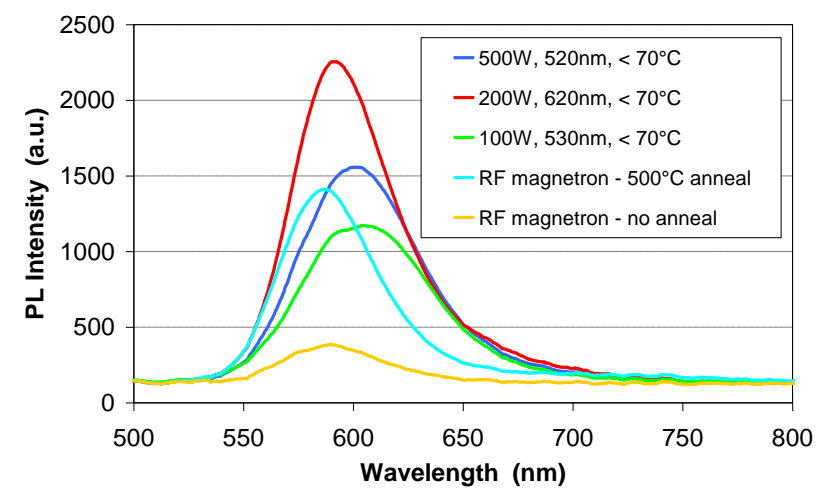

Figure 2 PL intensity as a function of target power for HiTUS ZnS:Mn films deposited with no substrate heating or post deposition annealing. The results of films deposited at $200{ }^{\circ} \mathrm{C}$ and post deposition annealed at $500{ }^{\circ} \mathrm{C}$ using RF magnetron sputtering have been included for comparison.

Following the successful deposition of $\mathrm{ZnS}: \mathrm{Mn}$ onto glass and $\mathrm{Si}$ substrates, single layers were also deposited onto Kapton $^{\mathrm{TM}}(50 \mu \mathrm{m})$, planarised polyethylene naphathalate (PEN - $125 \mu \mathrm{m})$ and polyethylene terephthalate (PET $-188 \mu \mathrm{m})$ using HiTUS. Coatings with a thickness of up to $1.0 \mu \mathrm{m}$ resulted in no deterioration of the flexible substrates.

The positive PL results indicate that these films should be excellent candidates for EL devices. Earlier work on ITO deposited using HiTUS identified this as a good material for the top and bottom contact electrodes due to its low resistivity (less than $4.0 \times 10^{-4} \Omega \mathrm{cm}$ ), high transmission and flexibility [2,5]. ITO films $200 \mathrm{~nm}$ thick deposited onto planarised PEN and PET have been shown to be flexible to a $1.0 \mathrm{~cm}$ bend radius with no subsequent increase in sheet resistance.

Dielectric layers of $\mathrm{Y}_{2} \mathrm{O}_{3}$ and $\mathrm{Ta}_{2} \mathrm{O}_{5}$ were also deposited onto glass and polymeric substrates with excellent transparency and breakdown properties, making possible the fabrication of completely transparent and flexible ACTFEL test devices.

3.1 ZnS:Mn XRD results XRD analysis of these films showed that they are highly crystalline exhibiting a major diffraction peak at a $2 \theta$ angle of $28.5^{\circ}$. Subsidiary 
peaks also occur at $59^{\circ}$ and $95^{\circ}$. Peak matching revealed that the probable structure is either cubic or cubic with small admixtures of hexagonal phase in line with previous reports [6]. The crystallite sizes, as calculated from the Scherer equation and the FWHM of the main peaks, are found to be $79 \mathrm{~nm}, 73 \mathrm{~nm}$ and $69 \mathrm{~nm}$ for target powers of $200 \mathrm{~W}, 500 \mathrm{~W}$ and $100 \mathrm{~W}$ respectively. The crystallite size of the films deposited at $200{ }^{\circ} \mathrm{C}$ and post deposition annealed at $500{ }^{\circ} \mathrm{C}$ using RF magnetron sputtering is significantly smaller at $16 \mathrm{~nm}$. This data is to form part of a further study looking at the correlation between crystallographic structure and PL response.

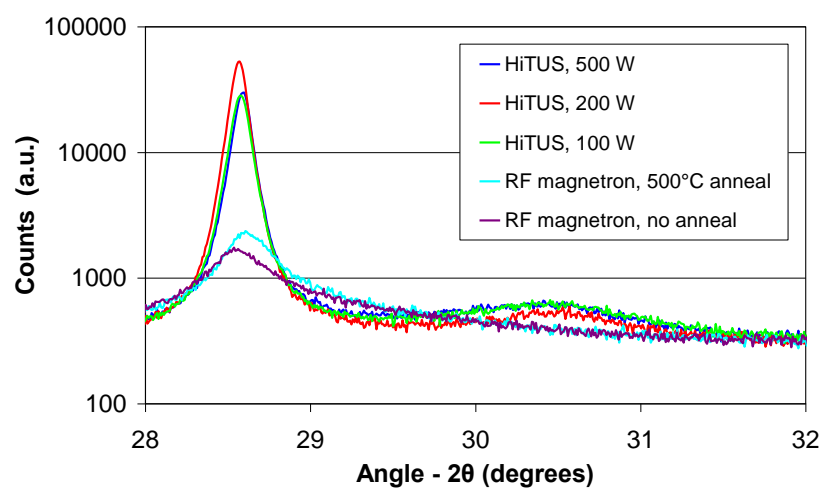

Figure 3 XRD peaks for single layer ZnS:Mn films deposited using HiTUS. The results of films deposited at $200{ }^{\circ} \mathrm{C}$ and post deposition annealed at $500{ }^{\circ} \mathrm{C}$ using RF magnetron sputtering are also included.

It is also clear from figure 3 that the optimum, as deposited, HiTUS films exhibit XRD peaks with higher intensity than $\mathrm{ZnS}: \mathrm{Mn}$ films deposited at $200{ }^{\circ} \mathrm{C}$ and post deposition annealed at $500{ }^{\circ} \mathrm{C}$ using RF magnetron sputtering. However, the HiTUS devices deposited with no substrate heating do not yield the same intense EL as the ones heated at $600{ }^{\circ} \mathrm{C}$. This possibly indicates that the substitution of the $\mathrm{Zn}$ with $\mathrm{Mn}$ ions in the host lattice is not occurring as preferentially as when the films are heated. Due to the decoupling of the ion density and ion energy, this could possibly be addressed by varying the RF power without affecting other deposition conditions. By increasing the RF power, and hence the plasma density, significant energy can be imparted to the growing film which could increase the probability of $\mathrm{Mn}$ ions substituting for $\mathrm{Zn}$ atoms in the $\mathrm{ZnS}$ lattice. This will form part of a further study to look at how we can improve the performance of the EL devices without needing to heat the substrates either during or post deposition.

4 Electroluminescence results The fabrication of ACTFEL test devices using both HiTUS and RF magnetron sputtering has been realised. The double insulating layer structure has been used for all devices with $\mathrm{Y}_{2} \mathrm{O}_{3}$ being used as the dielectric. Devices were initially deposited onto silicon substrates with ITO being used as the top contact. The possibility of making completely transparent de- vices on both glass and flexible substrate materials was then considered. The results in this section focus on a comparison of the two sputtering techniques to deposit working, EL devices on both glass and silicon substrates. Working EL structures were also deposited onto planarised PET using HiTUS. These results are presented elsewhere [1]. Due to the higher processing temperatures employed by RF magnetron sputtering, it was not possible to deposit devices onto plastic substrates using this technique.

4.1 EL devices deposited onto Si wafers Figure 4 shows the luminance as a function of applied peak to peak voltage (L-V) for an EL device deposited using RF magnetron sputtering. It is clear that heating the sample not only reduces the threshold voltage but also increases the maximum attainable luminance. This is to be expected as heating of the $\mathrm{ZnS}: \mathrm{Mn}$ layer results in a greater number of Mn ions finding their energetically favoured position as substitutes for the $\mathrm{Zn}$ atoms within the host lattice. In this position they readily contribute to the emission of light as active, luminescent centres. When located as interstitial defects they simply act as scattering centres for electrons. The effect of annealing the complete EL structure is that the hot electrons injected from the interface states are released at lower fields and hence the threshold voltage for EL emission occurs for lower voltages. Above the threshold voltage the gradient of the $\mathrm{L}-\mathrm{V}$ curve also becomes flatter for an anneal temperature of $600{ }^{\circ} \mathrm{C}$ indicating an increased energy distribution of interface states [7]. After post annealing in a vacuum at $600{ }^{\circ} \mathrm{C}$ the luminance is seen to increase to approximately $500 \mathrm{Cd} / \mathrm{m}^{2}$ at $750 \mathrm{~V}$.

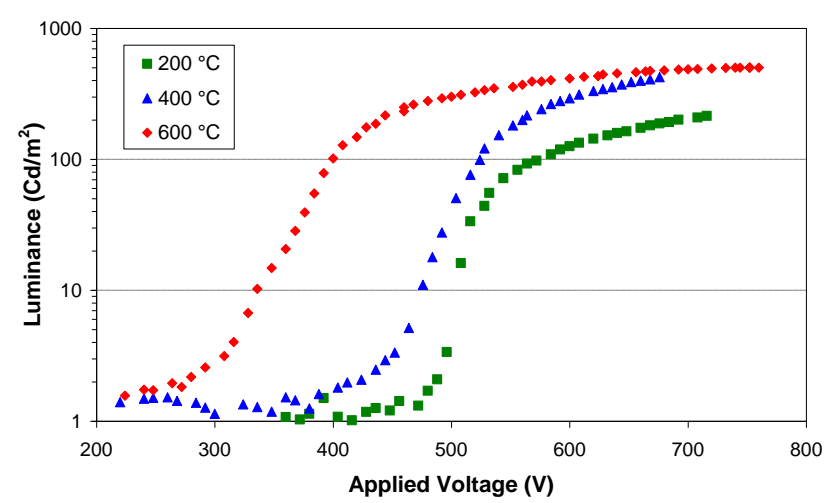

Figure 4 Luminance as a function of applied peak to peak voltage for an EL device on silicon fabricated using RF magnetron sputtering.

For comparison, the results of an equivalent device incorporating ITO and dielectric layers deposited using RF magnetron sputtering but ZnS:Mn deposited using HiTUS with no substrate heating are shown in figure 5. A maximum luminance of $740 \mathrm{Cd} / \mathrm{m}^{2}$ is now obtained for an applied peak to peak voltage of $690 \mathrm{~V}$. Of significance is the sharpness of the turn-on when the structure is heated to $600{ }^{\circ} \mathrm{C}$. This suggests that devices made with HiTUS 
$\mathrm{ZnS}: \mathrm{Mn}$ result in an interface region with a more concentrated distribution of trapped states. The increase in threshold voltage when increasing the anneal temperature from 400 to $600{ }^{\circ} \mathrm{C}$ is an interesting result that may imply a modification of the density of interface states via the removal of higher energy states (traps), or possibly a modification of the high field resistivity of the $\mathrm{ZnS}$ :Mn leading to increased threshold for impact excitation. This is to be investigated further. An increase in luminance of approximately $315 \mathrm{Cd} / \mathrm{m}^{2}$ for a rise in voltage of just $50 \mathrm{~V}$ is seen. The same rise in voltage for the devices fabricated using ZnS:Mn deposited by RF magnetron sputtering sees only a $17 \mathrm{Cd} / \mathrm{m}^{2}$ increase from 300 to $350 \mathrm{~V}$, the steepest section of the L-V curve. This makes the drive electronics potentially much easier and cheaper to implement for devices deposited using HiTUS ZnS:Mn.

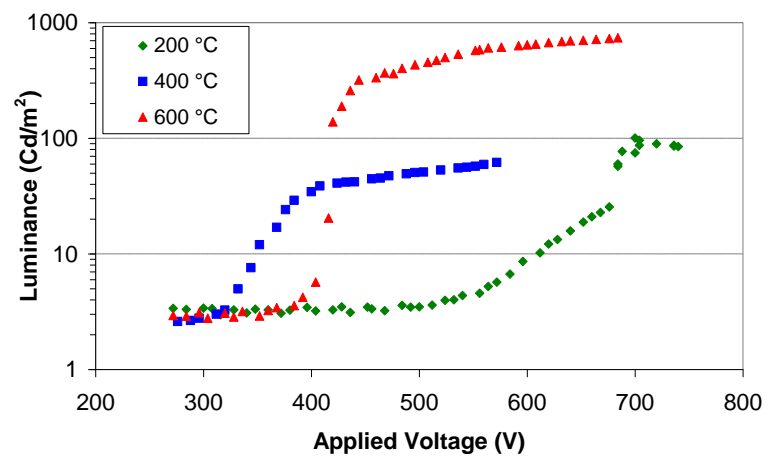

Figure 5 Luminance as a function of applied peak to peak voltage for an EL device on silicon fabricated using RF magnetron sputtering and HiTUS ZnS:Mn.

4.2 EL devices deposited onto glass substrates Completely transparent EL devices have been fabricated on glass substrates using an ITO back contact layer. The transmission of the devices at $595 \mathrm{~nm}$, taking the uncoated glass substrate as the reference, is approximately $96 \%$ and the average visible transmission from 400 to 750 $\mathrm{nm}$ is $87 \%$.

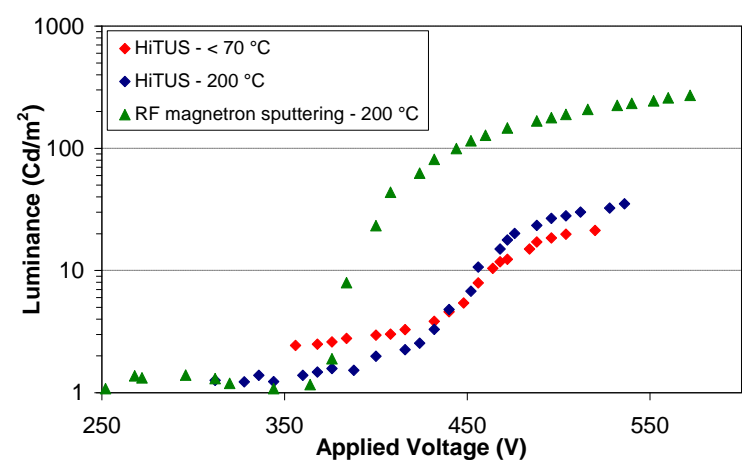

Figure 6 The luminance of a transparent EL structure deposited onto glass with no substrate heating or post deposition annealing using HiTUS. The effect of annealing at $200{ }^{\circ} \mathrm{C}$ is also shown, as are the results of magnetron sputtering at elevated substrate temperatures.
Figure 6 shows an L-V curve for a complete structure deposited using HiTUS, with no substrate heating or post deposition annealing. The effect of annealing at $200{ }^{\circ} \mathrm{C}$ is also shown although this results in little improvement of the device. For comparison, the results of post deposition annealing a device deposited at the substrate temperatures quoted in table 2 using RF magnetron sputtering is also shown. The maximum luminance is significantly higher with substrate heating. However, this obviously precludes the use of these devices for plastic applications. Devices deposited at ambient substrate temperature using RF magnetron sputtering exhibit no PL or EL and also breakdown very easily. It should be noted that the $\mathrm{Y}_{2} \mathrm{O}_{3}$ layer used in the HiTUS devices has not yet been optimised.

5 Conclusions ACTFEL devices have been deposited onto Si and glass substrates using two different sputtering techniques. Equivalent structures have been compared in terms of device performance for these two different technologies. Devices deposited onto silicon wafers using HiTUS ZnS:Mn are seen to offer a brighter maximum luminance as well as sharper turn-on characteristics despite being deposited with no substrate heating. Additionally, HiTUS allows for the deposition of working EL devices with no substrate heating or post deposition annealing; an achievement not possible with RF magnetron sputtering. The results presented thus far suggest that if the $\mathrm{Y}_{2} \mathrm{O}_{3}$ layers deposited using HiTUS can be optimised, ambient EL devices with comparable luminance to equivalent devices deposited using RF magnetron sputtering at elevated substrate temperatures could be possible.

\section{References}

[1] S. J. Wakeham, M. J. Thwaites, W. M. Cranton, C. Tsakonas and D. C. Koutsogeorgis, Eurodisplay 2009, Rome. Pending review.

[2] S. J. Wakeham, M. J. Thwaites, B. W. Holton, C. Tsakonas, W. M. Cranton, D. C. Koutsogeorgis, R. Ranson, $2^{\text {nd }}$ International Symposium on Transparent Conducting Oxides, Crete 2008. Accepted for publication. Available online TSF26149.

[3] Y. Hoshi and R. Ohki, Electrochemical Acta, vol 44, Issue 21-22, 1999, pp 3927-3932.

[4] G.Y. Yeom, J.A. Thornton and M.J. Kushner, Journal of Applied Physics, vol 65, Issue 10, 1989, pp 3825-3832.

[5] C. Tsakonas, W. M. Cranton, D. C. Koutsogeorgis, R. Ranson, S. J. Wakeham, B. Holton, M. J. Thwaites, 2nd International Symposium on Transparent Conducting Oxides, Crete 2008. Pending publication.

[6] E.A. Mastio, M.R Craven, W.M. Cranton, C.B. Thomas, M. Robino and E. Fogarassy, Journal of Applied Physics, vol 86, Issue 5, 1999, pp 2562-2570.

[7] W.M. Cranton PhD thesis, "Growth and characterisation of ZnS:Mn based laterally emitting thin film Electroluminescent device structures”, Bradford University, 1995. 